\title{
Conditions for the existence of Kelvin-Helmholtz instability in a CME
}

\author{
Andrés Páez ${ }^{1}$, Vera Jatenco-Pereira ${ }^{1}$, Diego Falceta-Gonçalves ${ }^{2}$ and \\ Merav Opher ${ }^{3}$ \\ ${ }^{1}$ Universidade de São Paulo, Instituto de Astronomia, Geofísica e Ciências Atmosféricas, \\ Rua do Matão 1226, São Paulo, SP, 05508-090, Brazil \\ email: andresspaez@usp.br \\ ${ }^{2}$ Universidade de São Paulo, Escola de Artes, Ciências e Humanidades, \\ Rua Arlindo Bettio 1000, São Paulo, SP, 03828-000, Brazil \\ email: dfalceta@usp.br \\ ${ }^{3}$ Astronomy Department, Boston University, \\ Boston, MA 02215, USA \\ email: mopher@bu.edu
}

\begin{abstract}
The presence of Kelvin-Helmholtz instability (KHI) in the sheaths of Coronal Mass Ejections (CMEs) has been proposed and observed by several authors in the literature. In the present work, we assume their existence and propose a method to constrain the local properties, like the CME magnetic field intensity for the development of KHI. We study a CME in the initiation phase interacting with the slow solar wind (Zone I) and with the fast solar wind (Zone II). Based on the theory of magnetic KHI proposed by Chandrasekhar (1961) we found the radial heliocentric interval for the KHI existence, in particular we constrain it with the CME magnetic field intensity. We conclude that KHI may exist in both CME Zones but it is perceived that Zone I is more appropriated for the KHI formation.
\end{abstract}

Keywords. Sun, Coronal mass ejection, Kelvin-Helmholtz instability.

\section{Introduction}

Coronal Mass Ejections (CMEs) are one of the strongest ejections $\left(10^{31}\right.$ ergs and $10^{15}$ g), originated at the solar surface. A normal CME has a three-part structure morphology: core, cavity and frontal loop (Illing \& Hundhausen 1985). A CME propagates with velocities of 20 to $3200 \mathrm{~km} / \mathrm{s}$ (Brueckner et al. 1995), expands with velocities approximately twice radial velocity (Rigozo, Dal Lago \& Nordeman 2011) and deflects in the interplanetary space with magnitude and direction depending mainly on the CME mass and velocity (Kay, Opher \& Evans 2015). The CME propagation allows shock wave formation ahead of the ejection, and the CME-sheath formation by the plasma compression behind the shock.

The Kelvin-Helmholtz instability (hereafter KHI), is a fast phenomenon in the boundary interface between two fluids developed mainly by the existence of a velocity gradient or velocity shear. The velocity shear provides kinetic energy to the boundary surface of the fluids allowing the formation of the rolled-up KH vortex. The KHI can be affected by the topology and magnitude of the magnetic field (Chandrasekhar 1961). A magnetic field parallel to the interface of the fluids is critical for the KHI development.

Foullon et al. (2011) analyzed the fast CME of November 3 of 2010 occurred near the southeast of the solar limb in the active region (AR) NOAA 11121 where is clear the formation of the flux rope. They considered the development of the KHI in the north flank of the ejection in distances of $150 \mathrm{Mm}$ above the solar surface. Foullon et al. (2013) 


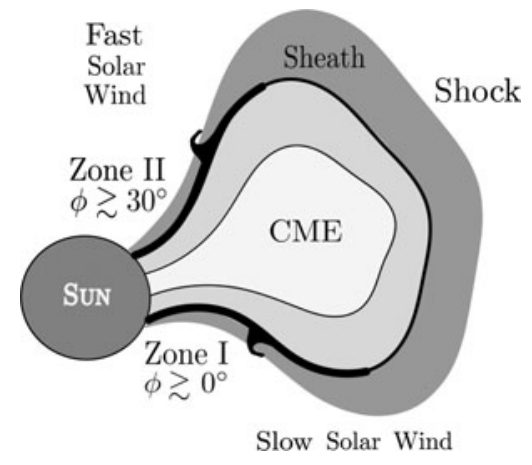

Figure 1. A cartoon showing the CME Zones I and II the regions of our study. Zone I represents the interaction with slow solar wind (latitudes $\phi \gtrsim 0^{\circ}$ ) and Zone II the interaction with the fast solar wind $\left(\phi \gtrsim 30^{\circ}\right)$. The shaded area represents the CME sheath.

made a spectral analysis to explore the CME plasma structure to corroborate the features observed by Foullon et al. (2011). Nykyri \& Foullon (2013) showed with 2.5-D magnetohydrodynamic (MHD) simulations the development of the KHI in the conditions of the ejecta of November 3 of 2010. With this work they showed the first calculus for determining the magnetic fields appropriated for the KHI development between the sheath and its CME in distances of the low corona. A similar situation observed by Foullon (2011) is shown in Figure 1. The regions of our study are represented by Zones I and II. The first one represents the interaction with slow solar wind (latitudes $\phi \gtrsim 0^{\circ}$ ) and Zone II the interaction with the fast solar wind $\left(\phi \gtrsim 30^{\circ}\right)$. The shaded area represents the CME sheath.

In this paper, we implement a method for finding the distances in which heliospheric distances the KHI exists. We study a CME in the initiation phase in interaction with the slow solar wind (Zone I) and with the fast solar wind (Zone II).

\section{Model}

We adopt the condition of existence of the magnetic KHI proposed by Chandrasekhar (1961) to the interaction between solar wind and a CME, in an infinitely thin sheath. We assumed a electron-proton plasma, with densities $\rho=\rho_{e}+\rho_{p}$, with the approximations, $\rho_{e} \approx n_{e} m_{e}, \rho_{p} \approx n_{p} m_{p}\left(m_{e}, m_{p}\right.$ are electron and proton masses), and quasi-neutrality $\mathrm{MHD}, n_{e} \approx n_{p} \approx n$ :

$$
\left(U_{S W}-U_{C M E}\right)^{2}>\frac{n_{S W}+n_{C M E}}{m_{p} n_{S W} n_{C M E}}\left(B_{S W}^{2}+B_{C M E}^{2}\right),
$$

where $U_{S W}, U_{C M E}, n_{S W}, n_{C M E}, B_{S W}$ and $B_{C M E}$ are solar wind and CME velocities, numerical densities and magnetic field, respectively.

We have constructed velocity functions for the solar wind slow and fast using a profile hyperbolic tangent, $U_{S W}^{\text {Slow, Fast }}(r)=\operatorname{atanh}[(r-1) / b]+d\left(\mathrm{~km} \mathrm{~s}^{-1}\right)($ Coles et al. 1991), with the transformation: $\tanh (r-1)$ to initiate at the solar surface. We adjust the constants $a, b$ and $d$ in there different points, the solar surface, $1 \mathrm{R}_{\odot}$ with $U_{S W}^{S l o w, F a s t}$ velocities taken from Harra et al. (2008), He et al. (2010), 3R $\odot$ from Quémerais et al. (2007), Antonucci, Dodero \& Giordano (2000) and $40 \mathrm{R}_{\odot}$, where we assume a constant value for the slow and fast solar wind. For the numerical densities of the slow and fast solar wind, we use mass conservation law where the values at the solar surface were taken from Fludra et al. (1999). For the magnetic field of the solar wind we use conservation of 
Table 1. Values for slow and fast solar wind velocities in three heliocentric distances $r=1,3$ and $40 \mathrm{R}_{\odot}$, values for the constants $a, b$ and d, values for the densities and for the magnetic fields at $r=1 R_{\odot}$.

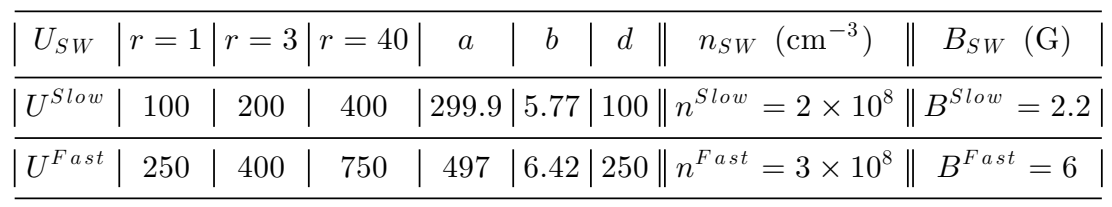

magnetic flux law where the values at the solar surface were evaluated from (Manchester et al. 2004) see Table 1.

For the existence of the KHI we are interested only in the Zones I and II where both magnetic fields of slow and fast solar wind and CME are parallel. In these situations we have a sheath with higher density, which we suppose to have a constant value of $n_{\text {sheath }}=10^{9} \mathrm{~cm}^{-3}$ (Hannah \& Kontar 2013).

\section{Results and Conclusions}

Constraint the intervals of heliocentric distances where de KHI can exists, $r_{K H}$, we have to find the values of velocities and densities for slow and fast solar wind and CME that satisfies the equation (2.1), linked with the value of CME magnetic field intensity. We found that in the Zone I the KHI can exists in the heliocentric distances of $0.15 \mathrm{R}_{\odot} \leqslant$ $r_{K H}^{S l o w} \leqslant 0,61 R_{\odot}$ linked to $1 \mathrm{G} \leqslant B_{C M E}^{S l o w} \leqslant 1.81 \mathrm{G}$. In Zone II the KHI can exists for distances $r_{K H}^{F a s t} \leqslant 1.41 \mathrm{R}_{\odot}$ linked to $1 \mathrm{G} \leqslant B_{C M E}^{F a s t} \leqslant 2.88 \mathrm{G}$. The interval for $r_{K H}^{S l o w}$ is in accordance with the results obtained by Foullon et al. (2011) and the values for $B_{C M E}^{S l o w}$ is in agreement with the work of Möstl, Temmer \& Veronig (2013).

\section{References}

Antonucci, E., Dodero, M. A., \& Giordano, S. 2000, Solar Phys., 197, 115

Brueckner, G. E., Howard, R. A., \& Koomen, M. J., et al. 1995, Solar Phys., 162, 357

Chandrasekhar, S. 1961, Hydrodynamic and hydromagnetic stability, International Series of Monographs on Physics, Oxford: Clarendon.

Coles, W. A., Esser, R., Lvhaug, U.-P., \& Markkanen, J. 1991, J. Geophys. Res., 96, 13849

Fludra, A., Del Zanna, G., Alexander, D., \& Bromage, B. J. I. 1999, J. Geophys. Res., 104, 9709

Foullon, C., Vernichte, E., Nakariakov, V. M., Nykyri, K., \& Farrugia, C. J. 2011, ApJ., 729, L8

Foullon, C., Vernichte, E., Nykyri, K., Aschwanden, M. J., \& Hannah, I. G. 2013, ApJ, 767, 170

Hannah, I. G. \& Kontar, E. P. 2013, A\& $A$, 553, A10

Harra, L. K., Sakao, T., \& Mandrini, C. H., et al. 2008, ApJ, 676, L147

He, J.-S., Marsch, E., Tu, C.-Y., Guo, L.-J., \& Tian, H. 2010, A\&A, 516, A14

Illing, R. M. E. \& Hundhausen, A. J. 1985, J. Geophys. Res., 90, 490

Kay, C., Opher, M., \& Evans, R. M. 2015, ApJ, 805, 168

Manchester, W. B., Gombosi, T. I., Roussev, I. de Zeeuw, D. L., Sokolov, I. V., Powell, K. G., Tóth, G., \& Opher, M. 2004, J. Geophys, Res., 109, 1102

Möstl, U. V., Temmer, M., \& Veronig, A. M. 2013, ApJ, 766, L12

Nykyri, K. \& Foullon, C. 2013, Geophys. Res. Lett., 40, 4154

Quémerais, E., Lallement, R., Koutroumpa, D., \& Lamy, P. 2007, ApJ, 667, 1229

Rigozo, N. R., Dal Lago, A., \& Nordeman, D. J. R. 2011, ApJ, 738, 107 